\title{
Targeting IRAK1 in T-Cell acute Iymphoblastic leukemia
}

\author{
Charles Dussiau ${ }^{1, *}$, Amélie Trinquand ${ }^{1, *}$, Ludovic Lhermitte ${ }^{1}$, Mehdi Latiri ${ }^{1}$, Mathieu \\ Simonin ${ }^{1}$, Agata Cieslak ${ }^{1}$, Nawel Bedjaoui ${ }^{1}$, Patrick Villarèse ${ }^{1}$, Els Verhoeyen ${ }^{2,3}$, \\ Hervé Dombret ${ }^{4}$, Norbert Ifrah ${ }^{5}$, Elizabeth Macintyre ${ }^{1}$, Vahid Asnafi ${ }^{1}$ \\ ${ }^{1}$ Université Paris Descartes Sorbonne Cité, Institut Necker-Enfants Malades (INEM), Institut National de Recherche Médicale \\ (INSERM) U1151, and Laboratory of Onco-Hematology, Assistance Publique-Hôpitaux de Paris (AP-HP), Hôpital Necker- \\ Enfants Malades, Paris, France \\ ${ }^{2}$ CIRI, EVIR Team, INSERM, U1111, CNRS, UMR5308, Université de Lyon-1, ENS de Lyon, Lyon, France \\ ${ }^{3}$ INSERM, U1065, C3M, Equipe "Contrôle Métabolique des Morts Cellulaires", Nice, France \\ ${ }^{4}$ University Paris 7, Hôpital Saint-Louis, AP-HP, Department of Hematology and Institut Universitaire d'Hématologie, EA, \\ Paris, France \\ ${ }^{5}$ PRES LUNAM, CHU Angers Service des Maladies du Sang et INSERM U 892, Angers, France \\ *These authors have contributed equally to this work
}

Correspondence to:

Vahid Asnafi, e-mail: vahid.asnafi@nck.aphp.fr

Keywords: T-ALL, IRAK1, kinases, therapeutic target

Received: December 12,2014 Accepted: May 20, $2015 \quad$ Published: June 01, 2015

\section{ABSTRACT}

T-cell acute lymphoblastic leukemia (T-ALL) represents expansion of cells arrested at specific stages of thymic development with the underlying genetic abnormality often determining the stage of maturation arrest. Although their outcome has been improved with current therapy, survival rates remain only around $50 \%$ at 5 years and patients may therefore benefit from specific targeted therapy. Interleukin receptor associated kinase 1 (IRAK1) is a ubiquitously expressed serine/threonine kinase that mediates signaling downstream to Toll-like (TLR) and Interleukin-1 Receptors (IL1R). Our data demonstrated that IRAK1 is overexpressed in all subtypes of T-ALL, compared to normal human thymic subpopulations, and is functional in T-ALL cell lines. Genetic knock-down of IRAK1 led to apoptosis, cell cycle disruption, diminished proliferation and reversal of corticosteroid resistance in T-ALL cell lines. However, pharmacological inhibition of IRAK1 using a small molecule inhibitor (IRAK1/4-Inh) only partially reproduced the results of the genetic knock-down. Altogether, our data suggest that IRAK1 is a candidate therapeutic target in T-ALL and highlight the requirement of next generation IRAK1 inhibitors.

\section{INTRODUCTION}

T-cell acute lymphoblastic leukemia (T-ALL) is an aggressive hematological malignancy resulting from the transformation of T-cell progenitors at various stages of development. They represent $10 \%$ of pediatric and $25 \%$ of adult ALL. Despite improvement in therapeutic protocols that cure nearly $80 \%$ children and $50 \%$ adults, relapse is common and the prognosis of relapsed T-ALL remains extremely poor $[1,2]$. T-ALL oncogenesis results from chromosomal rearrangements, somatic genetic mutations, aberrant oncogene expression and impairment of multiple signaling pathways involving kinases [3-7]. Interestingly, the use of several kinase inhibitors has been shown to be effective in T-ALL [8-10].Therefore, efficient evaluation of their potential therapeutic impact requires their use in subsets of patients that are most likely to benefit.

Interleukin receptor associated kinase (IRAK1) is a ubiquitously expressed serine/threonine kinase that mediates signal transduction downstream to Toll-like (TLRs) and Interleukin-1 Receptors (IL1R) [11]. Receptor activation results in phosphorylation of IRAK1 on threonine 209 which initiates recruitment of TRAF6, thus activating downstream NF- $\kappa$ B and MAPK pathways [12]. An oncogenic role for IRAK1 has recently been reported in myeloid cancers [13]. IRAK1 transcriptional expression has negative prognostic impact in Myelodysplasic syndromes (MDS) and acute myeloid leukemias (AML). Furthermore 
in vivo targeted inhibition of IRAK1 in a xenograft model of MDS demonstrated survival improvement [13, 14]. Oncogenic activation of the TLR/IL1R pathway is found in several B-cell lymphomas, often in conjunction with the MYD88 L265P gain of function mutation [15] and 100\% of primary effusion lymphoma harbor IRAK1 gain of function mutations leading to constitutive IRAK1 activation [16]. An IRAK1/4 inhibitor was also effective in MYD88 L265P mutated diffuse large B cell lymphoma (DLBCL) $[17,18]$.

We recently investigated the transcriptional expression of receptor and receptor-associated kinases in T-ALL by Taqman low density array (TLDA) [8]. We showed the overexpression of several kinases as compared to their normal thymic counterparts, demonstrating that exploration of the receptor kinome defines a rational strategy for testing kinase inhibition in T-ALL. These data showed that IRAK1 was strongly overexpressed in all categories of T-ALL so we sought to further explore the potential role of IRAK1 as a therapeutic target in T-ALL.

\section{RESULTS}

\section{IRAK1 is overexpressed and functional in T-ALL}

Transcriptional analysis of the expression level of 65 receptor and receptor-associated kinases in $32 \mathrm{~T}-\mathrm{ALL}$ (test series) and normal thymic subsets (cell-sorting described in Supplementary Figure S1) showed that IRAK1 was the most highly expressed kinase in all categories of T-ALL, regardless of the immunogenetic stage of arrest or underlying recurrent oncogenetic abnormalities, including Notch1 pathway mutations (Figure 1). We used qPCR to validate the transcriptional pattern of IRAK1 in sorted normal thymic subsets, in T-ALL cell lines, and in a large series of 177 independent (validation series) primary human T-ALL. This confirmed IRAK1 overexpression in T-ALL and cell lines as compared to normal thymus ( $p<0.01$, Figure 2A). IRAK1 transcript levels were slightly higher in most mature TCRab lineage thymic subpopulations as compared to immature and mature TCRgd subsets, without statistical significance (Figure 2A). No difference was observed between mature and immature T-ALL subtypes (Figure 2A) or oncogenic subtypes (not shown) suggesting ubiquitous oncogenic IRAK-1 deregulation in T-ALL, irrespective of stage of maturation arrest and/or oncogenic deregulation.

The IRAK1 protein was also widely expressed in cell lines and primary T-ALL blasts, with a trend to overexpression as compared to normal thymus (Figure 2B). IRAK1 was constitutively phosphorylated on residue T209 at variable levels in lymphoblastic T-cell lines and primary T-ALLs and to a lesser extent in normal human thymus (Figure 2B). In addition, IRAK1 T209phosphorylation increased over time in the Jurkat T-cell line upon IL-1 $\beta$ stimulation, suggesting a functional IRAK1 pathway (Figure 2C, left panel). Of note, the
IL-1 $\beta$-induced IRAK1 increased phosphorylation was specifically observed in cell lines harboring basal IRAK1 phosphorylation (Jurkat and ALL-SIL T-cell lines) but not in HPB-ALL and DND-41, which express IRAK1 proteins without basal phosphorylation (Figure $2 \mathrm{C}$ right panel).

Taken together, these data suggest that IRAK1 is robustly expressed in T-ALL at both transcriptional and protein levels, and remains functional in at least a significant subset of cases.

\section{Knock-down of IRAK1 induces apoptosis and disrupts cell cycle in T-ALL}

To test whether IRAK1 is required for T-ALL survival, we transduced short hairpin RNAs (shRNA) targeting IRAK1 into T-ALL cell lines. Two independent shRNA (543 and 544) significantly downregulated IRAK1 protein expression in several cell lines (representative data for Jurkat, Figure 3A). Depletion of IRAK1 with both shRNA induced a dramatic decrease in T-cell proliferation (Figure 3B) and increase in apoptosis and cell cycle disruption by increasing the $\mathrm{G} 0 / \mathrm{G} 1$ and decreasing the $\mathrm{S}$ phase cells in both HPB-ALL and Jurkat cell-lines (Figure 3C and 3D).

\section{Knock-down of IRAK1 reverses resistance to corticosteroids in Jurkat}

Resistance to corticosteroids remains one of the most challenging issues in ALL therapy. As previously reported [19], the Jurkat cell line is resistant to corticosteroids (Figure 4A). To assess whether knock-down of IRAK1 could reverse this resistance, we tested the effects of dexamethasone in Jurkat after genetic IRAK1 inactivation. Dexamethasone induced apoptosis was significantly increased in cells transduced with IRAK1 shRNA as compared to control cells (Figure 4A). A similar effect was observed on cell proliferation (Figure 4B). In order to confirm these data, we then performed assays with a broader range of dexamethasone doses tested on a p-IRAK positive (Jurkat) and a p-IRAK negative (HPB-ALL) T-ALL cell line with simultaneous genetic knockdown of IRAK-1 using shRNA544 (Figure 4C). These data demonstrated that the corticosteroid response reversion is specifically observed in p-IRAK1 positive cells (Figure 4C). Altogether these data support that knockdown of IRAK1 could sensitize corticosteroid resistant p-IRAK1 positive T-ALL to dexamethasone therapy.

\section{Pharmacological inhibition only partially reproduces the effects of genetic knock-down}

We then submitted Jurkat, to increasing doses of the previously reported pharmacological inhibitor of IRAK1 (IRAK1/4-Inh) [20, 21]. A significant decrease in phosphorylation of IRAK1 was observed after $48 \mathrm{~h}$ 


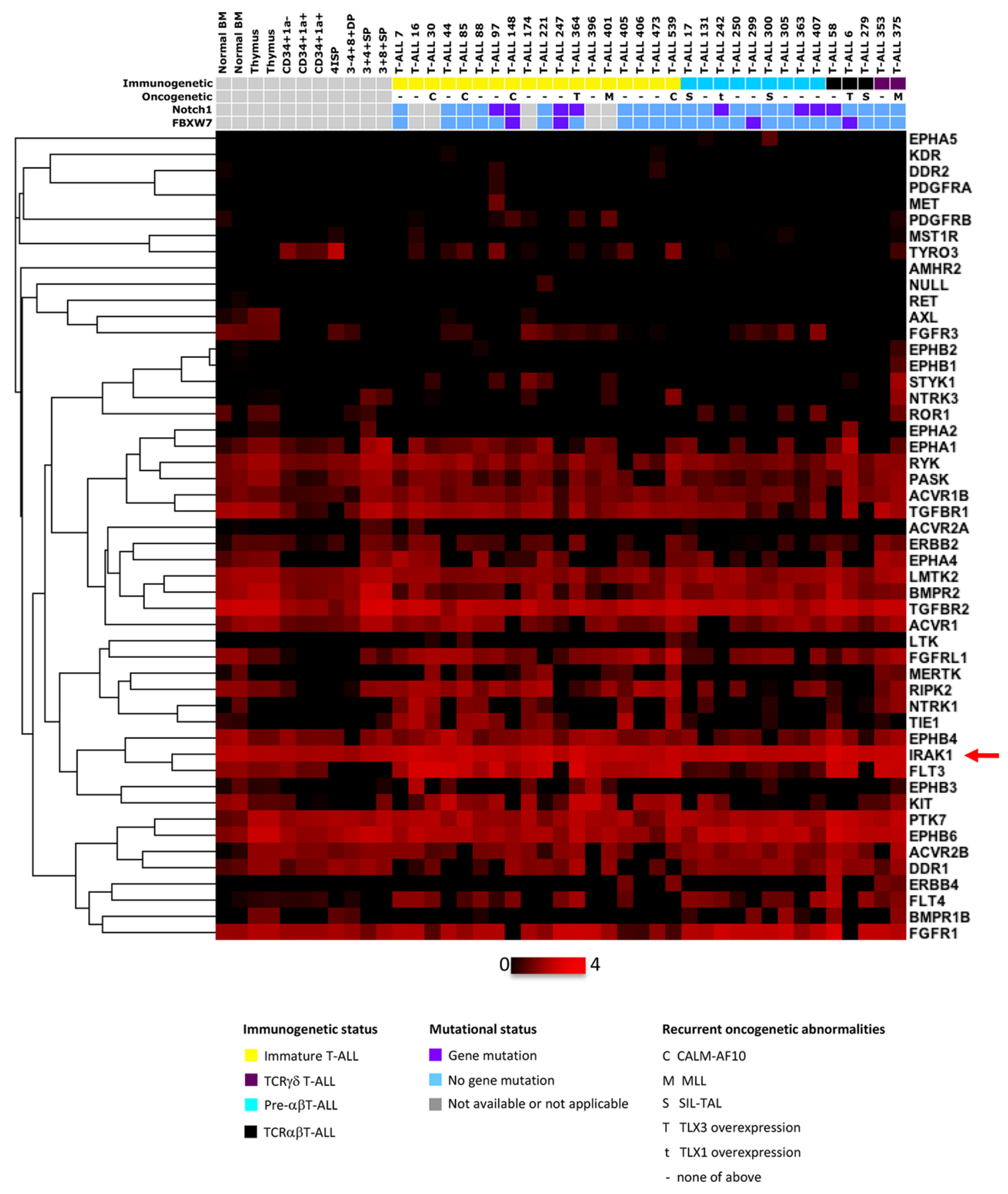

Figure 1: Kinases expression profiles of human T-ALL samples and thymic subpopulations. Transcriptional expression of major kinase receptors and receptor associated kinases in normal and malignant immature T-cells. Thymic subpopulations and T-ALL samples are displayed in a supervised classification model and ordered according to their immunogenetic status. Non-expressed (receptor)kinases are not shown. 4ISP, CD4 immature single positive; DP TCR-, CD4/CD8 double positive surface TCR negative; DP TCR+, CD4/ CD8 double positive surface TCR positive; SP4, mature CD4 single positive; SP8, mature CD8 single positive.

treatment, demonstrating a biochemical effect of the inhibitor (Figure 5A). However, the apoptotic effect and the decrease in proliferation induced in the Jurkat cell-line upon IRAK1 inhibition were not as dramatic with the pharmacologic inhibition as with the genetic knockdown (Figure 5B and 5C). However, these effects remained significant compared to the HPBALL p-IRAK1 negative cell-line (Figure 5D) and are consistent with previously reported data on MDS/ AML [13]. To confirm these data, we finally performed pharmacological inhibition of IRAK1 in two primary T-ALL samples (T-ALL3 and T-ALL4). Similarly, 
A

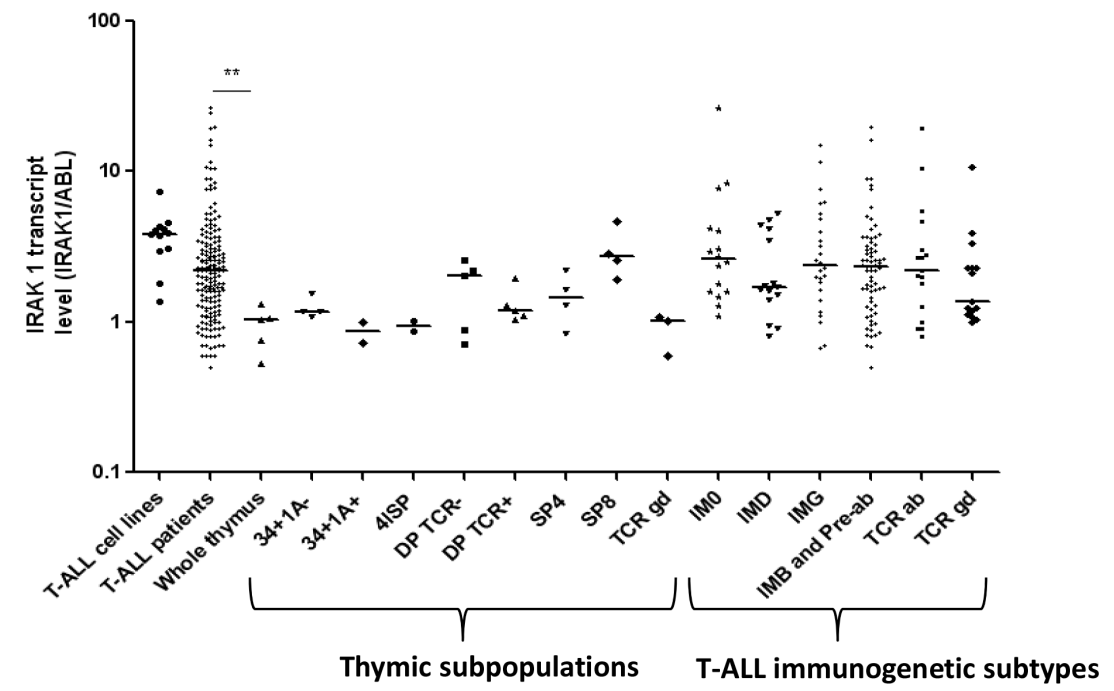

B
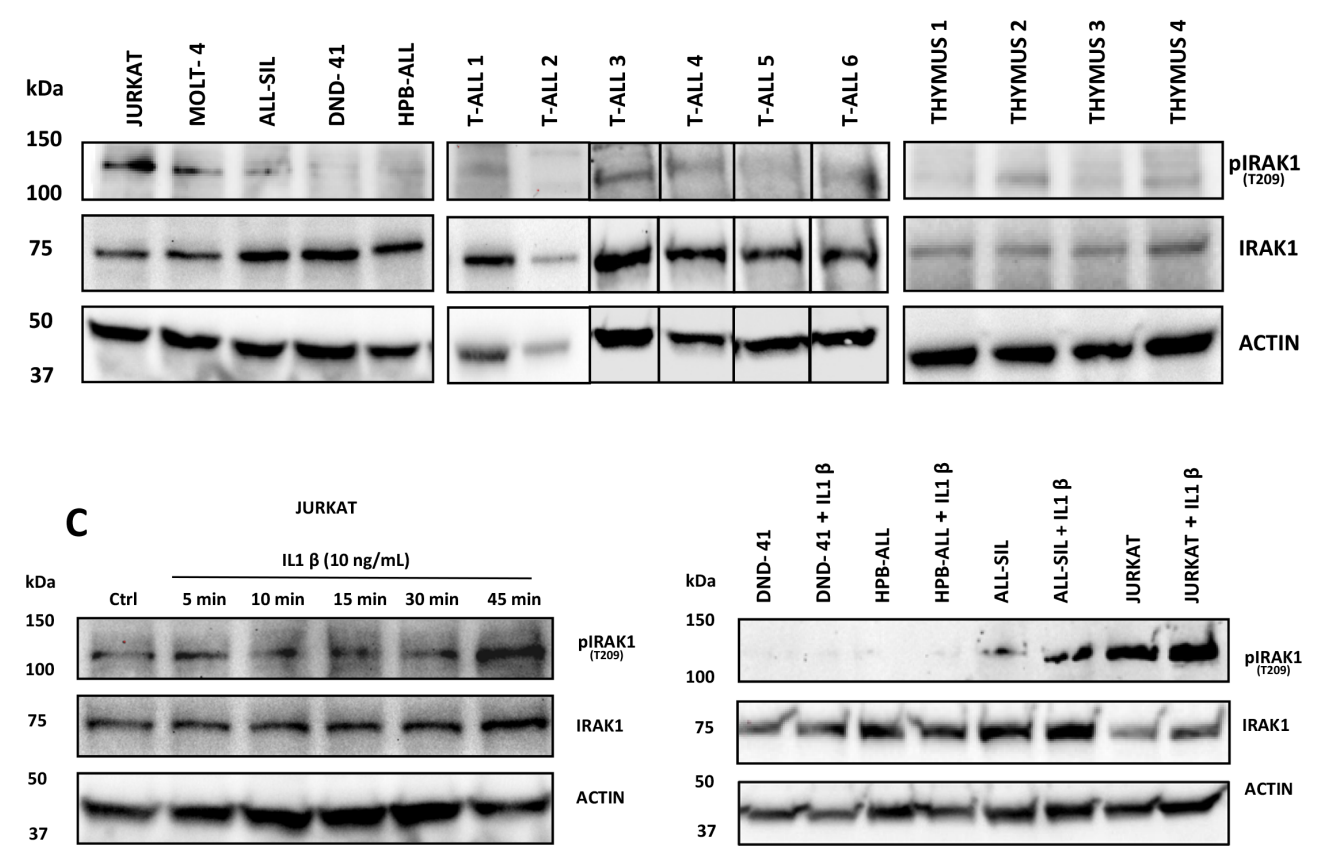

Figure 2: IRAK1 is overexpressed and functional in T-ALL. A. qRT-PCR: IRAK1 transcriptional expression is shown in T-ALL according to TCR status, and in thymic subsets. B. IRAK1 protein expression and phosphorylation were assessed by western blot on T-ALL cell lines, primary T-ALL blasts and normal thymus. C. Left panel: Activation of IRAK1 pathway at different time upon IL1 $\beta$ stimulation in the Jurkat cell line. Right panel: Activation of IRAK1 pathway after 45 min treatment withIL1 $\beta$ (10 ng/mL) in T-ALL cell lines. 4ISP, CD4 immature single positive; DP TCR-, CD4/CD8 double positive surface TCR negative; DP TCR+, CD4/CD8 double positive surface TCR positive; SP4, mature CD4 single positive; SP8, mature CD8 single positive; IM0, immature with germline TCR loci; IMB, immature with TCR $\beta$ rearrangement; Pre-ab, cTCR $\beta$ expressing T-ALL [31].

we observed a significant increase in cell apoptosis of primary T-ALL samples upon pharmacological inhibition of IRAK1/4 (Figure 5E). Taken together, genetic knockdown and, to a lesser extent, pharmacological inhibition of IRAK1 both resulted in decreased proliferation and induction of apoptosis in the Jurkat cell-line and primary T-ALL blasts.

\section{DISCUSSION}

Improving prognosis remains a therapeutic challenge in T-ALL. Kinases have recently been shown to be druggable targets in T-ALL, particularly in Early T-precursor (ETP)-ALL, a very immature subtype with myeloid features which is associated with a high risk 
A

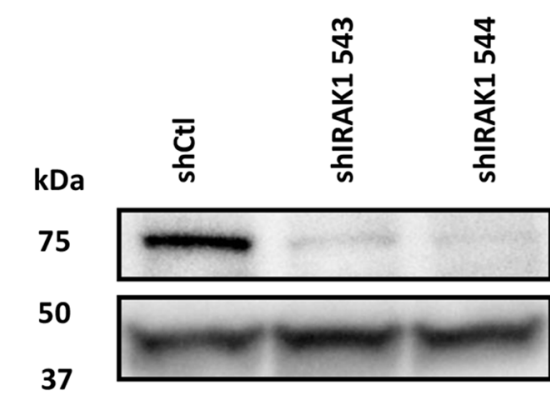

IRAK1

ACTIN
B

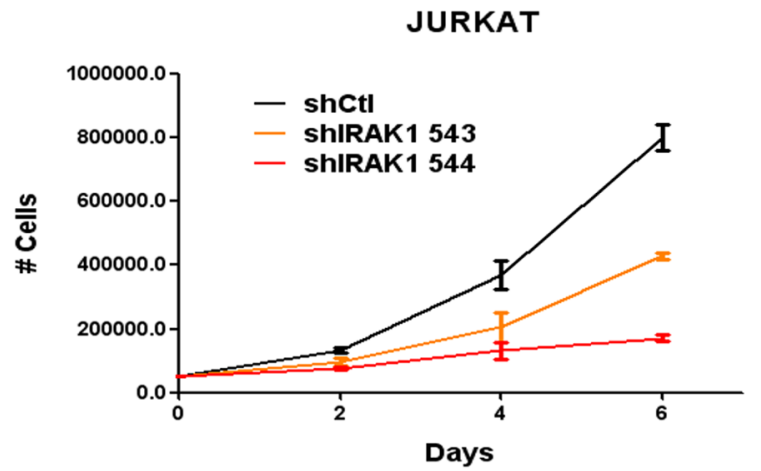

HPB-ALL

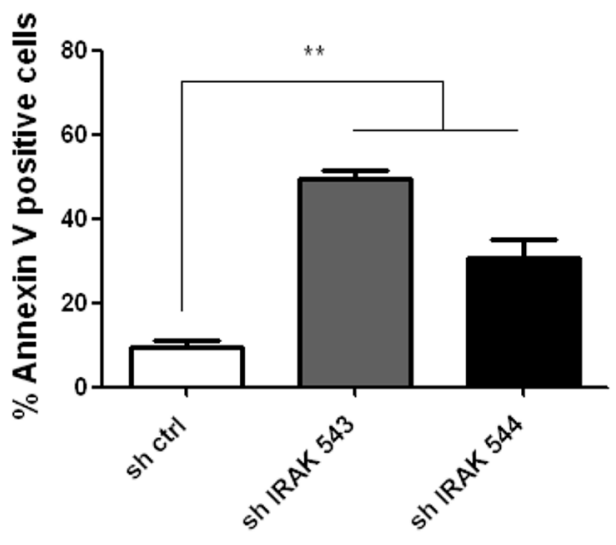

HPB-ALL

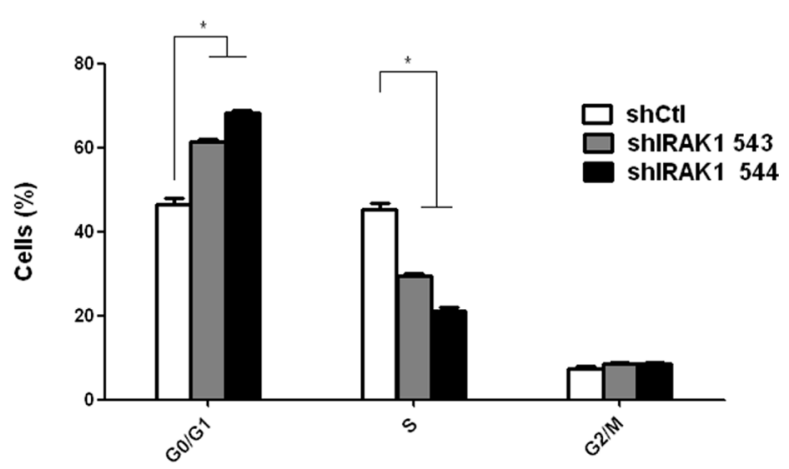

Figure 3: Genetic knock-down of IRAK1 induces apoptosis and disrupts cell cycle. A. Genetic knockdown of IRAK1 was confirmed by western blotting in Jurkat cells expressing a control or shIRAK1-expressing lentiviral vector B. Viable cell growth was assayed by trypan blue exclusion for up to 6 days. Data are represented as mean $+/-$ SEM. C. Annexin V/IP staining was assessed in Jurkat and HPB-ALL cells by flow cytometry after transduction with shRNA-expressing lentiviral vectors. D. Cell cycle analysis of Jurkat and HPB-ALL cells after transduction was performed with Edu/7-AAD incorporation. 
A

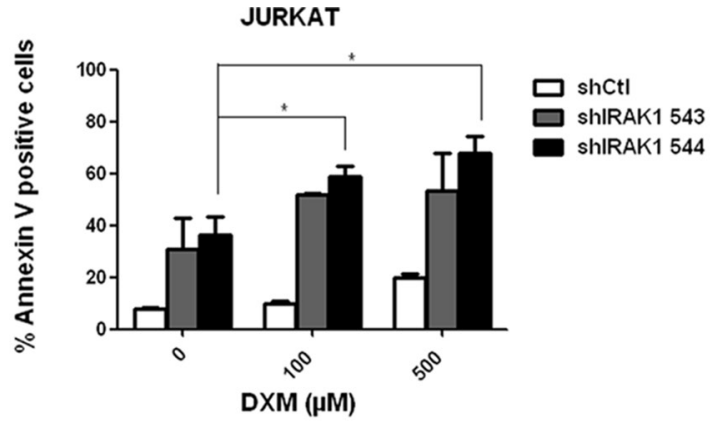

B

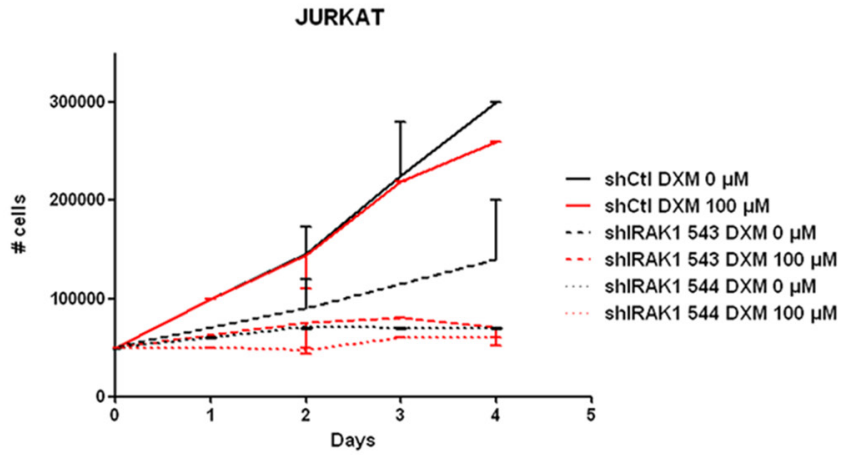

C

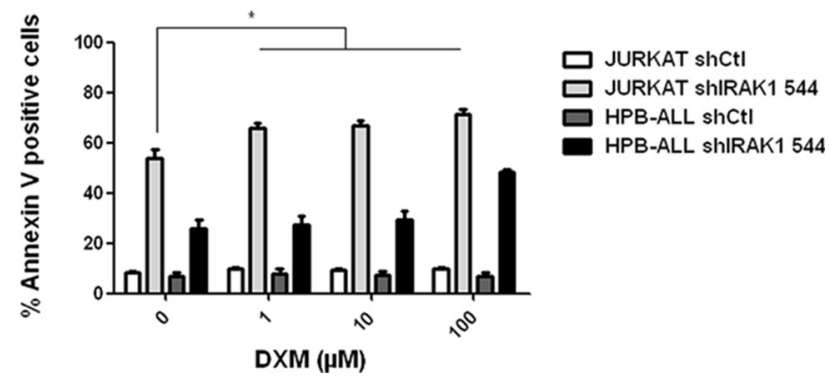

Figure 4: Genetic knockdown of IRAK1 reverses corticoresistance in Jurkat. A. AnnexinV/IP staining was assessed in Jurkat after transduction with shRNA-expressing lentiviral vector and after $72 \mathrm{~h}$ treatment with dexamethasone $(0-100-500 \mu \mathrm{M})$. B. Viable cell growth was assayed by trypan blue exclusion after transduction with shRNA-expressing lentiviral vector and treatment with dexamethasone $(0-100 \mu \mathrm{M})$ for up to 4 days. Data are represented as mean $+/-$ SEM. C. AnnexinV/IP staining was assessed after transduction with shRNAexpressing lentiviral vector in jurkat (white) and HPB-ALL (grey) and after $72 \mathrm{~h}$ treatment with dexamethasone (0-1-10-100 $\mu \mathrm{M})$ on Jurkat (light grey) and HPB-ALL (black) cell-lines. DXM, dexamethasone.

of induction failure or relapse and a poor outcome [22]. Indeed, kinases play a key role in signaling pathways downstream to cytokine receptors that are important in T-ALL and central to ETP ALL oncogenesis [3]. We have now identified IRAK1 as a novel candidate kinase for targeted therapy in T-ALL, including ETP-ALL. IRAK1/4 inhibitor was originally developed for autoimmune and inflammatory diseases [20], and was effective in-vitro and in-vivo in hematological malignancies (MDS, AML, DLBCL) that overactivate the IRAK1 pathway $[13,18]$, albeit to a lesser extent. These data in T-ALL underline the requirement of new and more efficient IRAK1 inhibitor for clinical use in leukemia therapeutics.

Our data support that inhibition of IRAK1 activity result in corticoresistance reversion, only in the context of constitutive activation of IRAK1. The unexpected observation that pharmacological inhibition of IRAK1 could not result in comparable cytotoxicity to genetic knock-down of IRAK1 raises the possibility that the cellular oncogenic effects of IRAK1 may not be entirely mediated through its kinase domain. There is precedent for this in alternative disease models, such as the FLT3 receptor which displays both kinase-dependent and independent effects. One mechanism accounting for primary resistance to pharmacological FLT3 inhibition appears to be related to the maintenance of kinaseindependent effects of FLT3 upon TKD exposure, with active recruitment of signaling molecules on Grb2 and downstream overexpression of MCL1, thereby maintaining oncogenic FLT3 activity [23]. Interestingly, previous studies showed that kinase-dead IRAK1 mutants maintain IL1-mediated NF-kB and 
A

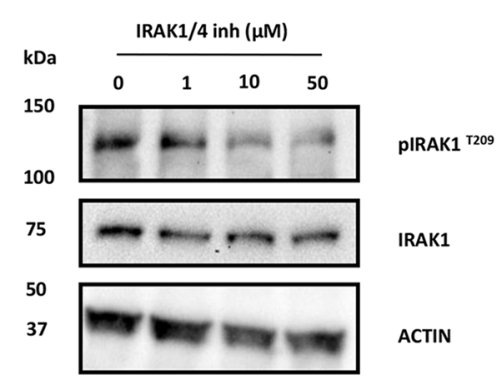

C

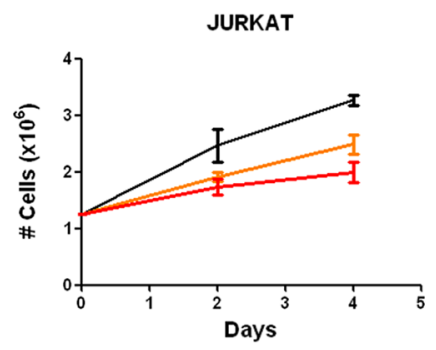

$\mathbf{E}$

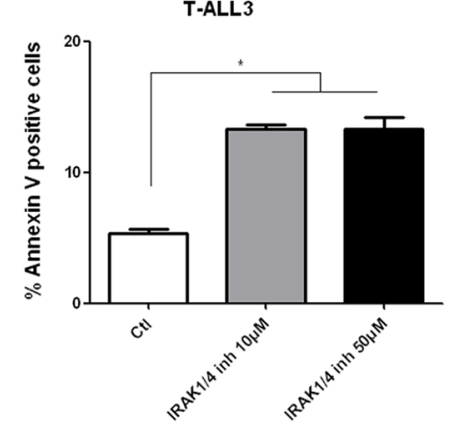

T-ALL 4

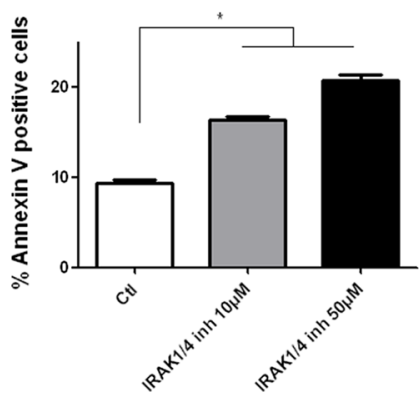

B

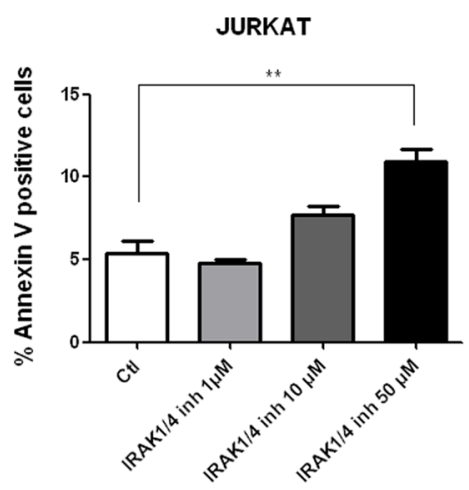

D

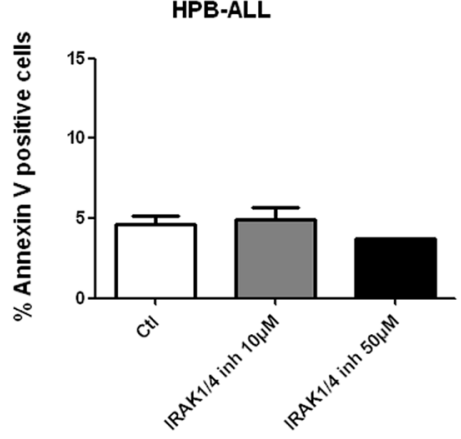

T-ALL3

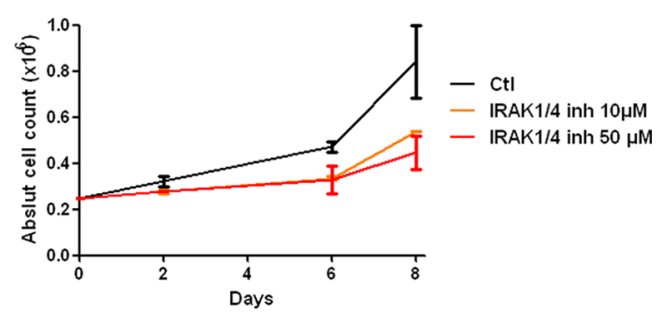

T-ALL4

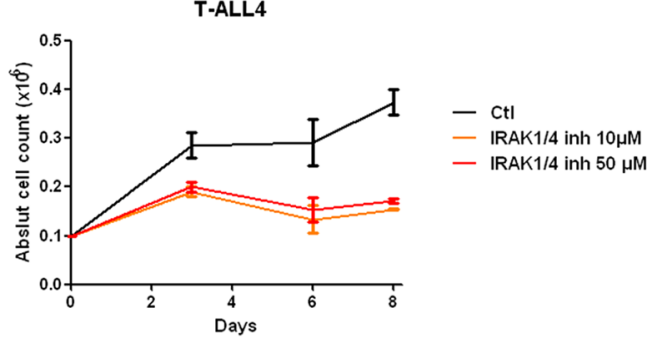

Figure 5: Effect of IRAK1/4-inh on T-ALL cell lines. A. IRAK1 and pIRAK ${ }^{\mathrm{T} 209}$ were evaluated by western blotting in Jurkat cells after $48 \mathrm{~h}$ treatment with IRAK1/4 inhibitor B-E. AnnexinV/IP staining was performed after $72 \mathrm{~h}$ treatment with IRAK1/4 inhibitor in Jurkat, HPB-ALL cells and in primary T-ALL blasts from 2 patients (C-E) Viable cell growth of Jurkat and in primary T-ALL blasts from 2 patients was assayed by trypan blue exclusion in the presence of IRAK1/4 inhibitor $(0-10-50 \mu \mathrm{M})$. Data are represented as mean $+/-$ SEM.

MAPK activation, suggesting that the kinase domain of IRAK1 may be dispensable for the activation of some physiological downstream signaling mediators [24-26]. Whether the present discrepancy between genetic and pharmacological inhibition results from kinase-independent signaling remains to be addressed. However, the exact sequence of molecular events following IRAK1 activation is not fully deciphered and kinase-related and kinase-independent effects remain controversial [27]. 
We identified deregulation of IRAK1 in all T-ALL subsets but the molecular mechanism(s) of overexpression and constitutive activation in hematological malignancies has not been addressed so far. It may result from posttranscriptional deregulation by miRNA such as miR-146, which targets IRAK1 mRNA [28]. The role of IRAK1 in normal thymopoïesis and in T-ALL pathogenesis should be further investigated. It has been shown that the NF- $\kappa$ B pathway is activated and can be targeted in T-ALL [29]. In this context, targeting IRAK1 may represent a new approach to inhibit the NF- $\kappa \mathrm{B}$ pathway preferentially in leukemic compared to normal lymphoid precursors.

In conclusion, our study identifies IRAK1 as a potential novel target in T-ALL, particularly in corticoresistant T-ALL and ETP-ALL but optimal therapeutic intervention will require development of next generation IRAK1 inhibitors and their preliminary evaluation in in-vivo tests and mouse xenograft models of T-ALL.

\section{MATERIALS AND METHODS}

\section{Patients}

Diagnostic peripheral blood or bone marrow samples from 177 adults with T-ALL were analyzed after informed consent was obtained at diagnosis according to the Declaration of Helsinki. Patients were included in the GRAALL03/05 trials, both were registered at ClinicalTrials.gov (GRAALL-2003, NCT00222027; GRAALL-2005, NCT00327678).

Normal thymic samples were obtained from children undergoing cardiac surgery at the Necker-Enfants Malades Hospital, with informed consent from the parents.

\section{Molecular analysis (Taqman Low Density Array and real-time quantitative PCR)}

Quantification of kinase expression was performed on quality-controlled cDNA using predesigned TaqMan Low Density microfluidic cards (TLDA Human Protein Kinase Array; Applied Biosystems). Samples with GAPDH cycle thresholds superior to 21 were excluded from analysis. Each probe value was normalized with respect to GAPDH copy number and analyzed with a $\Delta 13$ cycle threshold to discard background noise.

IRAK1 transcript expression was quantified by real-time quantitative PCR in an unselected series of 177 T-ALLs with available cDNA.

\section{Cell lines and culture conditions}

The JURKAT, MOLT-4, ALL-SIL, DND-41, and HPB-ALL T cell lines were grown in RPMI 1640 supplemented with $50 \mu \mathrm{g} / \mathrm{mL}$ streptomycin, 50 IU penicillin, $2 \mathrm{mM}$ L-glutamine and $20 \%$ fetal bovine serum $\left(10 \%\right.$ for JURKAT). Cell cultures were maintained at $37^{\circ} \mathrm{C}$ in a humidified atmosphere containing $5 \% \mathrm{CO}_{2}$. Primary T-ALL were co-cultured on OP9-DL1 in a $\alpha$-MEM media supplemented with 20\% FBS (Hyclone ; Thermo Fisher Scientific), $50 \mu \mathrm{g} / \mathrm{ml}$ streptomycin , 50 IU penicillin and recombinant human cytokines hFLT3-L ( $5 \mathrm{ng} / \mathrm{mL})$, hIL-7 (2 ng/mL) and hSCF (10 ng/mL) (Miltenyi).

\section{Reagents}

The IRAK1 inhibitor (IRAK1/4 inhibitor or IRAKInh; Amgen Inc.) was purchased from Merck Millipore (Ref 407602). Recombinant human IL1- $\beta$ was purchased from Miltenyi (Ref 130-093-897).

\section{Apoptosis, cell cycle analysis and growth curves}

Apoptosis was assessed by flow cytometry using Annexin V APC (and propidium iodide (BD-Pharmingen, San Jose, CA, USA) staining.

Cell cycle analysis was performed by flow cytometry using EdU/7-AAD staining purchased from Invitrogen, as per manufacturer recommendations.

Cell viability was measured with trypan blue exclusion using a Hemocytometeror an automated cell counter (CASY, Roche Diagnostics).

\section{Western blotting}

T-ALL cell lines and primary T-ALL cells at diagnosis were washed in PBS and lysed in HNTG buffer (50mM Hepes $\mathrm{pH} 7.4,150 \mathrm{mM} \mathrm{NaCl}, 50 \mathrm{mM} \mathrm{NaF}$, $1 \mathrm{mM}$ EDTA, 1\% Triton, $10 \%$ glycerol, $1.5 \mathrm{mM} \mathrm{MgCl}_{2}$ ) supplemented with protease and phosphatase inhibitors (Halt Protease and Phosphatase Inhibitor Cocktail, Thermo scientific). Protein was separated by $7.5 \%$ SDS-PAGE (MiniProtean, Biorad) and transferred onto nitrocellulose membranes. After blocking $1 \mathrm{~h}$ in TBST 5\% BSA, membranes were incubated overnight at $4{ }^{\circ} \mathrm{C}$ with the first $\mathrm{Ab}$ in blocking buffer. Primary Abs used were: antipIRAK1 ${ }^{\text {T209 }}$ (1/500, Assay Biotech) anti-IRAK1 (1/1000, Cell Signaling) and anti-ACTIN (1/1000, Santa Cruz). After washing with TBST, the membrane was incubated for $30 \mathrm{~min}$ at room temperature with the appropriate secondary $\mathrm{Ab}$ coupled to HRP in blocking buffer. The signal was detected using the WestDura supersignal or WestFemto supersignal kit (GE Healthcare Bio-Sciences).

\section{Knock-down of IRAK1 with shRNA lentivirus}

Two independent and validated pLKO.1-shIRAK1 constructs developed by Rhyasen et al [13] were used (TRCN0000000543 and TRCN0000000544).

\section{Statistical analysis}

All monoparametric measurement comparisons were determined using the Mann-Whitney test (PRISM 
software, GraphPad, La Jolla, CA, USA). All Tests were two-sided, with $p<0.05$ considered statistically significant)

Hierarchical classifications were performed using the Pearson's correlation coefficient as a similarity metric with an average linkage algorithm as described [30].

\section{ACKNOWLEDGMENTS}

This work was supported by grants to Necker laboratory from the "Association Laurette Fugain", the "Comité Départemental de la Ligue Contre le Cancer" and the Institut National du Cancer. Samples were collected and processed by the AP-HP "Direction de Recherche Clinique" Tumor Bank at Necker-Enfants Malades.

CD was supported by Assistance Publique des Hôpitaux de Paris (AP- HP).

\section{REFERENCES}

1. Inaba $\mathrm{H}$, Greaves $\mathrm{M}$, Mullighan $\mathrm{CG}$. Acute lymphoblastic leukaemia. Lancet. 2013; 381:1943-1955.

2. Bassan R, Hoelzer D. Modern therapy of acute lymphoblastic leukemia. J Clin Oncol. 2011; 29:532-543.

3. Zhang J, Ding L, Holmfeldt L, Wu G, Heatley SL, Payne-Turner D, Easton J, Chen X, Wang J, Rusch M, Lu C, Chen SC, Wei L, Collins-Underwood JR, Ma J, Roberts KG, et al. The genetic basis of early T-cell precursor acute lymphoblastic leukaemia. Nature. 2012; 481:157-163.

4. Bandapalli OR, Schuessele S, Kunz JB, Rausch T, Stutz AM, Tal N, Geron I, Gershman N, Izraeli S, Eilers J, Vaezipour N, Kirschner-Schwabe R, Hof J, von Stackelberg A, Schrappe M, Stanulla M, et al. The activating STAT5B N642H mutation is a common abnormality in pediatric T-cell acute lymphoblastic leukemia and confers a higher risk of relapse. Haematologica. 2014; 99:e188-92.

5. Van Vlierberghe P, Ferrando A. The molecular basis of $\mathrm{T}$ cell acute lymphoblastic leukemia. The Journal of clinical investigation. 2012; 122:3398-3406.

6. Sarmento LM, Povoa V, Nascimento R, Real G, Antunes I, Martins LR, Moita C, Alves PM, Abecasis M, Moita LF, Parkhouse RM, Meijerink JP, Barata JT. CHK1 overexpression in T-cell acute lymphoblastic leukemia is essential for proliferation and survival by preventing excessive replication stress. Oncogene. 2015;34:2978-90.

7. Kontro M, Kuusanmaki H, Eldfors S, Burmeister $\mathrm{T}$, Andersson EI, Bruserud O, Brummendorf TH, Edgren H, Gjertsen BT, Itala-Remes M, Lagstrom S, Lohi O, Lundan T, Marti JM, Majumder MM, Parsons A, et al. Novel activating STAT5B mutations as putative drivers of T-cell acute lymphoblastic leukemia. Leukemia. 2014; 28:1738-1742.

8. Lhermitte L, Ben Abdelali R, Villarese P, Bedjaoui N, Guillemot V, Trinquand A, Libura M, Bedin AS, Petit A,
Dombret H, Leverger G, Ifrah N, Hermine O, Macintyre E, Asnafi V. Receptor kinase profiles identify a rationale for multitarget kinase inhibition in immature T-ALL. Leukemia. 2013; 27:305-314.

9. Buontempo F, Orsini E, Martins LR, Antunes I, Lonetti A, Chiarini F, Tabellini G, Evangelisti C, Evangelisti C, Melchionda F, Pession A, Bertaina A, Locatelli F, McCubrey JA, Cappellini A, Barata JT, et al. Cytotoxic activity of the casein kinase 2 inhibitor CX-4945 against T-cell acute lymphoblastic leukemia: targeting the unfolded protein response signaling. Leukemia. 2014; 28:543-553.

10. Lonetti A, Antunes IL, Chiarini F, Orsini E, Buontempo F, Ricci F, Tazzari PL, Pagliaro P, Melchionda F, Pession A, Bertaina A, Locatelli F, McCubrey JA, Barata JT, Martelli AM. Activity of the pan-class I phosphoinositide 3-kinase inhibitor NVP-BKM120 in T-cell acute lymphoblastic leukemia. Leukemia. 2014; 28:1196-1206.

11. Cao Z, Henzel WJ, Gao X. IRAK: a kinase associated with the interleukin-1 receptor. Science. New York, NY: 1996; 271:1128-1131.

12. Flannery S, Bowie AG. The interleukin-1 receptorassociated kinases: critical regulators of innate immune signalling. Biochemical pharmacology. 2010; 80:1981-1991.

13. Rhyasen GW, Bolanos L, Fang J, Jerez A, Wunderlich M, Rigolino C, Mathews L, Ferrer M, Southall N, Guha R, Keller J, Thomas C, Beverly LJ, Cortelezzi A, Oliva EN, Cuzzola M, et al. Targeting IRAK1 as a therapeutic approach for myelodysplastic syndrome. Cancer cell. 2013; 24:90-104.

14. Beverly LJ, Starczynowski DT. IRAK1: oncotarget in MDS and AML. Oncotarget. 2014; 5:1699-1700.

15. Jimenez C, Sebastian E, Chillon MC, Giraldo P, Mariano Hernandez J, Escalante F, Gonzalez-Lopez TJ, Aguilera C, de Coca AG, Murillo I, Alcoceba M, Balanzategui A, Sarasquete ME, Corral R, Marin LA, Paiva B, et al. MYD88 L265P is a marker highly characteristic of, but not restricted to, Waldenstrom's macroglobulinemia. Leukemia. 2013; 27:1722-1728.

16. Yang D, Chen W, Xiong J, Sherrod CJ, Henry DH, Dittmer DP. Interleukin 1 receptor-associated kinase 1 (IRAK1) mutation is a common, essential driver for Kaposi sarcoma herpesvirus lymphoma. Proc Natl Acad Sci U S A. 2014;111:E4762-8.

17. Rhyasen GW, Bolanos L, Starczynowski DT. Differential IRAK signaling in hematologic malignancies. Experimental hematology. 2013; 41:1005-1007.

18. Ngo VN, Young RM, Schmitz R, Jhavar S, Xiao W, Lim KH, Kohlhammer $\mathrm{H}$, Xu W, Yang Y, Zhao H, Shaffer AL, Romesser P, Wright G, Powell J, Rosenwald A, Muller-Hermelink HK, et al. Oncogenically active MYD88 mutations in human lymphoma. Nature. 2011; 470:115-119.

19. Riml S, Schmidt S, Ausserlechner MJ, Geley S, Kofler R. Glucocorticoid receptor heterozygosity combined with lack 
of receptor auto-induction causes glucocorticoid resistance in Jurkat acute lymphoblastic leukemia cells. Cell death and differentiation. 2004; 11:S65-72.

20. Powers JP, Li S, Jaen JC, Liu J, Walker NP, Wang Z, Wesche H. Discovery and initial SAR of inhibitors of interleukin-1 receptor-associated kinase-4. Bioorganic \& medicinal chemistry letters. 2006; 16:2842-2845.

21. Wang Z, Wesche H, Stevens T, Walker N, Yeh WC. IRAK-4 inhibitors for inflammation. Current topics in medicinal chemistry. 2009; 9:724-737.

22. Coustan-Smith E, Mullighan CG, Onciu M, Behm FG, Raimondi SC, Pei D, Cheng C, Su X, Rubnitz JE, Basso G, Biondi A, Pui CH, Downing JR, Campana D. Early T-cell precursor leukaemia: a subtype of very high-risk acute lymphoblastic leukaemia. The Lancet. 2009; 10:147-156.

23. Breitenbuecher F, Markova B, Kasper S, Carius B, Stauder T, Bohmer FD, Masson K, Ronnstrand L, Huber C, Kindler T, Fischer T. A novel molecular mechanism of primary resistance to FLT3-kinase inhibitors in AML. Blood. 2009; 113:4063-4073.

24. Knop J, Martin MU. Effects of IL-1 receptor-associated kinase (IRAK) expression on IL-1 signaling are independent of its kinase activity. FEBS letters. 1999; 448:81-85.

25. Li X, Commane M, Jiang Z, Stark GR. IL-1-induced NFkappa B and c-Jun N-terminal kinase (JNK) activation diverge at IL-1 receptor-associated kinase (IRAK). Proceedings of the National Academy of Sciences of the United States of America. 2001; 98:4461-4465.

26. Maschera B, Ray K, Burns K, Volpe F. Overexpression of an enzymically inactive interleukin-1-receptor-associated kinase activates nuclear factor-kappaB. The Biochemical journal. 1999; 339:227-231.

27. Moynagh PN. The Pellino family: IRAK E3 ligases with emerging roles in innate immune signalling. Trends in immunology. 2009; 30:33-42.

28. Taganov KD, Boldin MP, Chang KJ, Baltimore D. NF-kappaB-dependent induction of microRNA miR146, an inhibitor targeted to signaling proteins of innate immune responses. Proceedings of the National Academy of Sciences of the United States of America. 2006; 103:12481-12486.

29. Vilimas T, Mascarenhas J, Palomero T, Mandal M, Buonamici S, Meng F, Thompson B, Spaulding C, Macaroun S, Alegre ML, Kee BL, Ferrando A, Miele L, Aifantis I. Targeting the NF-kappaB signaling pathway in Notch1-induced T-cell leukemia. Nature medicine. 2007; 13:70-77.

30. Eisen MB, Spellman PT, Brown PO, Botstein D. Cluster analysis and display of genome-wide expression patterns. Proceedings of the National Academy of Sciences of the United States of America. 1998; 95:14863-14868.

31. Asnafi V, Beldjord K, Boulanger E, Comba B, Le Tutour P, Estienne MH, Davi F, Landman-Parker J, Quartier P, Buzyn A, Delabesse E, Valensi F, Macintyre E. Analysis of TCR, pT alpha, and RAG-1 in T-acute lymphoblastic leukemias improves understanding of early human T-lymphoid lineage commitment. Blood. 2003; 101:2693-2703. 